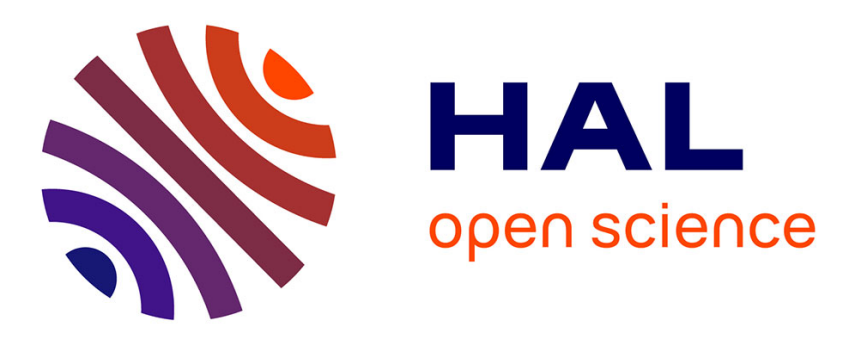

\title{
A homogenized cerebrospinal fluid model for diffuse optical tomography in the neonatal head
}

Stephanie Lohrengel, Farah Oumri, Stéphanie Salmon, Fabrice Wallois, Mahdi Mahmoudzadeh

\section{- To cite this version:}

Stephanie Lohrengel, Farah Oumri, Stéphanie Salmon, Fabrice Wallois, Mahdi Mahmoudzadeh. A homogenized cerebrospinal fluid model for diffuse optical tomography in the neonatal head. International Journal for Numerical Methods in Biomedical Engineering, 2021, 10.1002/cnm.3538 . hal-03144383

\author{
HAL Id: hal-03144383 \\ https://hal.science/hal-03144383
}

Submitted on 17 Feb 2021

HAL is a multi-disciplinary open access archive for the deposit and dissemination of scientific research documents, whether they are published or not. The documents may come from teaching and research institutions in France or abroad, or from public or private research centers.
L'archive ouverte pluridisciplinaire HAL, est destinée au dépôt et à la diffusion de documents scientifiques de niveau recherche, publiés ou non, émanant des établissements d'enseignement et de recherche français ou étrangers, des laboratoires publics ou privés. 


\title{
A homogenized CSF model for diffusive optical tomography in the neonatal head
}

\author{
Stephanie Lohrengel* $\quad$ Farah Oumri* $\quad$ Stéphanie Salmon*
}

February 17, 2021

\begin{abstract}
A new model for optical tomography in the neonatal brain is presented that takes into account the presence of arachnoid trabeculæ in the cerebrospinal fluid (CSF). It is known that the classical diffusion approximation for light propagation is at the limit of validity in the CSF layer due to the low values of the absorption and scattering coefficients. The new model is obtained by the diffusion approximation of the homogenized radiative transfer equation and is rigorously justified. Numerical results in two and three dimensions attest for the improved sensitivity of the new model to the presence of perturbations in the brain layer.
\end{abstract}

\section{Introduction.}

Near infrared (NIR) optical tomography is a method of medical imaging based on the absorption and scattering of light in the near infrared scale by biological tissues, and in particular those of the brain. This non-invasive and non-irradiating technique can be used for bedside monitoring and is thus particularly suitable for newborns or premature babies. A head cup is placed on the children's head, equipped with optodes which serve both as light sources and detectors. After passing through the various tissues of the head, the amount of light arriving at the detector is measured. This measurement constitutes the observable quantity and makes it possible to reconstruct changes in the optical parameters. The latter account for changes in hemoglobin concentration during cerebral activity or are used as indicators for pathological cerebral blood flow as for example intracranial hemorrhage (see [13] and references herein).

A large amount of papers deal with the mathematical modeling of optical tomography in general tissues (see e.g. $[4,5,27,12,8]$ ). Monte Carlo simulation is a popular method that describes light transport at the photon level. Its main drawback is the low resolution that implies the need of a huge number of photons in order to get results that are numerically stable. In our context, the radiative transfer equation (RTE) yields a valid model for photon transport. However, its discretization in a 3D setting is still costly due to the high number of variables ( 3 for space, 3 for the direction, 1 for the time). Therefore, the diffusion equation is widely used as an approximation of the RTE.

However, it is well known that the quality of the diffusion approximation depends on the ratio between the involved optical parameters which are the absorption and the scattering coefficient and several papers state that the diffusion approximation is not valid in cerebrospinal fluid (CSF) [20, 21, 24]. In order to overcome the difficult question of simulating light propagation in the CSF region, some authors suggest a radiosity-diffusion model that couples a model based on geometric optics in CSF with the diffusion approximation in the other regions. The model is shown to be well-posed [21] and numerical results in a 3D spherical domain attest its efficiency in dealing with void regions. In a realistic head model obtained from MR and CT images, the method could however be difficult to implement due to the nonlocal boundary conditions on the diffusion/void-interfaces. Indeed, the accurate segmentation of these interfaces is much more difficult than segmentation of the 3D regions occupied by the different tissues.

There is an inherent difficulty of detecting inclusions surrounded by a CSF region: due to the optical properties, photons in CSF have very small probability to be deviated from their trajectory. The CSF region thus acts as a wave guide and many photons arrive far from the source point without meeting the inclusions in the inner layers. This phenomenon can be observed in simulations based on the RTE or

\footnotetext{
* Laboratoire de Mathématiques LMR CNRS UMR 9008, Université de Reims Champagne Ardenne, Moulin de la Housse - B.P. 1039, 51687 Reims Cedex 2, France. Corresponding author: stephanie.lohrengel@univ-reims.fr

This work has received funding from the French National Research Agency (grant ANR-15-CE23-0009, MAIA project).
} 
on Monte Carlo codes. However, NIR spectroscopy and optical tomography were proven to be able to retrieve useful information from the recorded signals, even in the presence of CSF [13, 16]. The drawback is the high computational cost of Monte Carlo based simulations.

In this paper, we develop a new model for optical tomography based entirely on the diffusion approximation. The main idea is to take into account the presence of arachnoid trabeculæ (AT) among CSF. Arachnoid trabeculæ are thin filaments that connect the two inner meninges, arachnoid mater and pia mater. In [24], the results based on Monte Carlo simulations in a CSF layer filled with small cylindric regions of AT are in good agreement with experimental results if the CSF incorporates about $15 \%$ discrete scatterers. However, the simulations need a mesh that distinguishes between CSF and AT which have a very small cross section (about $0.1[\mathrm{~mm}]$ ). Consequently, the computational cost is much higher than for Monte Carlo simulations with homogeneous CSF. The resolution of the associated inverse problem, i.e. three-dimensional reconstruction of optical parameters, seems thus prohibited. Here, we apply homogenization techniques in order to substitute the two-phase layer by an equivalent homogeneous layer.

The paper is organized as follows. In Section 2, we make precise the classical diffusion equation that is used as an approximation of the RTE. We discuss the modeling of the source term as well as well as the choice of a parameter set for the optical parameters of the newborn's head. The comparison between measurements with and without the presence of a perturbation illustrate the lack of sensitivity of the classical model in the presence of the CSF layer. Section 3 is devoted to the description of the new model and we explain in which way homogenization has to be performed in order to get an improved diffusion model. The mathematical justification of the homogenization procedure is precised with the help of a result of [14] on homogenization of transport equations. Numerical results in two and three dimensions illustrate the improvements provided by the new model. Finally, in Section 4, we present a local sensitivity analysis via the concept of Gâteaux derivative for the new model.

\section{The classical model of diffusive optical tomography.}

\subsection{The radiative transfer equation.}

Any mathematical model of optical tomography is based on a model for light transport. In our context where the wavelength of light in the near infrared (NIR) range is small compared with the characteristic distances of the human head, the radiative transfer equation (RTE) yields a valid model for photon transport.

We denote by $\mu_{s}$ and $\mu_{a}$, respectively, the scattering and absorption coefficient of the medium in which light propagation occurs. The unknown of the RTE is the specific intensity of radiation $I(t, \mathbf{x}, \mathbf{s})$ which, at time $t$ and at a point $\mathbf{x}$, is defined by the radiation energy transferred by photons per unit steradian in the direction $\mathbf{s}$. Denote by $f\left(\mathbf{s}, \mathbf{s}^{\prime}\right)$ the phase function that describes the probability that a photon arriving by direction $\mathbf{s}$ will be scattered in direction $\mathbf{s}^{\prime}$. We thus have

$$
\int_{\mathbb{S}} f\left(\mathbf{s}, \mathbf{s}^{\prime}\right) d \mathbf{s}^{\prime}=4 \pi
$$

where the integral is taken on the unit sphere $\mathbb{S}$ of $\mathbb{R}^{3}$. In the sequel, we shall assume that the phase function is symmetric in the two variables.

Then, the principle of energy conservation leads to the following integro-differential equation, called radiative transfer equation:

$$
\frac{1}{c} \partial_{t} I(t, \mathbf{x}, \mathbf{s})+\mathbf{s} \cdot \nabla I(t, \mathbf{x}, \mathbf{s})+\mu_{t} I(t, \mathbf{x}, \mathbf{s})=\frac{\mu_{s}}{4 \pi} \int_{\mathbb{S}} f\left(\mathbf{s}, \mathbf{s}^{\prime}\right) I\left(t, \mathbf{x}, \mathbf{s}^{\prime}\right) d \mathbf{s}^{\prime}+q(t, \mathbf{x}, \mathbf{s}) .
$$

Here, $\mu_{t}=\mu_{s}+\mu_{a}$ denotes the total attenuation coefficient and $q$ models the light source. $c$ is the speed of light which is related to $c_{0}$, the speed of light in vacuum, through the refractive index $n=c_{0} / c>1$. We refer to [2] for a general introduction to transport and diffusion theory and to [5] for a discussion of the RTE model in the context of light propagation and appropriate boundary conditions. The numerical resolution of the RTE is computationally expensive because of the high number of variables (3 for the space variable, 3 for the directions). Discretization can be performed by means of finite difference or finite element methods, discontinous Galerkin schemes or Monte Carlo approaches [28, 30, 10, 22]. In real life applications, where the number of unknowns may be extremely high (typically about 600000 elements and 100000 nodes [6]), the use of the RTE in an inverse problem that needs the computation of a large number of forward solutions, may thus be prohibited with regard to the computational cost. 
The diffusion approximation (DA) is therefore widely used as an approximation for the RTE. Several methods exist to derive the diffusion equation from RTE, among which the $P_{N}$ approximation that is based on the expansion of the intensity $I(\cdot, \cdot, \mathbf{s})$ in terms of spherical harmonics, and an aysmptotic analysis in terms of a small scaling factor $\varepsilon \ll 1$ that measures the ratio between a (large) scattering coefficient $\mu_{s}$ and a (small) absorption coefficient $\mu_{a}$.

For the sake of completeness, we recall the main features of both approaches and refer the reader to $[2,5]$ and references therein for further details.

\subsubsection{The diffusion equation as $P_{1}$ approximation of RTE}

We introduce the following two quantities derived from the specific intensity $I$ as the moments of order 0 and 1 , respectively, with respect to the direction $\mathbf{s}$ : the diffuse photon density

$$
\Phi(t, \mathbf{x})=\int_{\mathbb{S}} I\left(t, \mathbf{x}, \mathbf{s}^{\prime}\right) d \mathbf{s}^{\prime}
$$

and the diffuse photon flux

$$
\mathbf{J}(t, \mathbf{x})=\int_{\mathbb{S}} I\left(t, \mathbf{x}, \mathbf{s}^{\prime}\right) \mathbf{s}^{\prime} d \mathbf{s}^{\prime}
$$

The following relations can be obtained from the developpement in terms of spherical harmonics truncated at order 1:

$$
\begin{aligned}
I(t, \mathbf{x}, \mathbf{s}) & =\frac{1}{4 \pi}(\Phi(t, \mathbf{x})+3 \mathbf{J}(t, \mathbf{x}) \cdot \mathbf{s}) \\
q(t, \mathbf{x}, \mathbf{s}) & =\frac{1}{4 \pi}\left(q_{0}(t, \mathbf{x})+3 \mathbf{q}_{1}(t, \mathbf{x}) \cdot \mathbf{s}\right)
\end{aligned}
$$

Integrating the RTE over the sphere $\mathbb{S}$ then yields

$$
\frac{1}{c} \partial_{t} \Phi(t, \mathbf{x})+\operatorname{div} \mathbf{J}(t, \mathbf{x})+\mu_{a} \Phi(t, \mathbf{x})=q_{0}(t, \mathbf{x})
$$

In the same way, taking the scalar product of RTE with $\mathbf{s}$ and integrating over $\mathbb{S}$ yields

$$
\frac{1}{c} \partial_{t} \mathbf{J}(t, \mathbf{x})+\frac{1}{3} \nabla \Phi(t, \mathbf{x})+\left(\mu_{a}+\mu_{s}^{\prime}\right) \mathbf{J}(t, \mathbf{x})=\mathbf{q}_{1}(t, \mathbf{x})
$$

where $\mu_{s}^{\prime}=(1-g) \mu_{s}$ denotes the reduced scattering coefficient which depends on the anisotropy factor $g$ defined by

$$
g=\frac{1}{4 \pi} \int_{\mathbb{S}} \mathbf{s} \cdot \mathbf{s}^{\prime} f\left(\mathbf{s}, \mathbf{s}^{\prime}\right) d \mathbf{s}^{\prime} .
$$

The following assumptions allow to retrieve the diffusion equation in terms of the unknown $\Phi$ :

- the source of light is isotropic:

$$
\mathbf{q}_{1}(t, \mathbf{x})=0,
$$

- the time derivative of the photon flux is small compared to the attenuated flux:

$$
\frac{1}{c} \partial_{t} \mathbf{J}(t, \mathbf{x}) \ll\left(\mu_{a}+\mu_{s}^{\prime}\right) \mathbf{J}(t, \mathbf{x}) .
$$

Under the assumptions (8), the photon flux derives from the scalar potential $\Phi$ that satisfies the following diffusion equation:

$$
\frac{1}{c} \partial_{t} \Phi(t, \mathbf{x})-\operatorname{div}(\kappa \nabla \Phi(t, \mathbf{x}))+\mu_{a} \Phi(t, \mathbf{x})=q_{0}(t, \mathbf{x})
$$

where the diffusion coefficient $\kappa$ is given by

$$
\kappa=\frac{1}{3\left(\mu_{a}+\mu_{s}^{\prime}\right)} .
$$




\subsubsection{Asymptotic analysis of the RTE.}

The principle of asymptotic analysis is the scaling of the RTE with the help of a small parameter $\varepsilon$. We aim to consider a large time scale as well as an absorption coefficient that is small compared to the scattering coefficient. Hence, we let

$$
\mu_{a}=\varepsilon \hat{\mu}_{a}, \mu_{s}=\frac{\hat{\mu}_{s}}{\varepsilon},
$$

with reference quantities $\hat{\mu}_{a}, \hat{\mu}_{s}$ of order 1 , and

$$
I(t, \mathbf{x}, \mathbf{s})=I_{\varepsilon}(\varepsilon t, \mathbf{x}, \mathbf{s})
$$

with a function $I_{\varepsilon}=I_{\varepsilon}(\hat{t}, \mathbf{x}, \mathbf{s})$ such that $\partial_{\hat{t}} I_{\varepsilon}=\mathcal{O}(1)$ [2]. The function $I_{\varepsilon}$ satisfies the RTE with coefficients $\hat{\mu}_{a}$ and $\hat{\mu}_{s}$ and is formally expanded in a series of powers of $\varepsilon$ :

$$
I_{\varepsilon}(\hat{t}, \mathbf{x}, \mathbf{s})=\frac{1}{4 \pi} \sum_{k=0}^{\infty} \varepsilon^{k} I_{k}(\hat{t}, \mathbf{x}, \mathbf{s}) .
$$

Identification of the powers of $\varepsilon$ shows at order 0 that $I_{0}$ is independent from $\mathbf{s}$,

$$
I_{0}=I_{0}(t, \mathbf{x}) .
$$

In absence of a source term $q$, it may be shown from the analysis of the equations at order 1 and 2 that $I_{0}$ satisfies the following diffusion equation depending on the scaled coefficients

$$
\frac{1}{c} \partial_{\hat{t}} I_{0}(\hat{t}, \mathbf{x})-\operatorname{div}\left(\hat{\kappa} \nabla I_{0}(\hat{t}, \mathbf{x})\right)+\hat{\mu}_{a} L I_{0}(t, \mathbf{x})=0,
$$

where

$$
\hat{\kappa}=\frac{1}{3 \hat{\mu}_{s}^{\prime}} .
$$

Notice that definition (14) of the diffusion coefficient is asymptotically equivalent to the former one (10). Indeed, in terms of the physical coefficients $\mu_{a}$ and $\mu_{s}^{\prime}$, we have

$$
\frac{1}{3\left(\mu_{a}+\mu_{s}^{\prime}\right)}=\frac{1}{3\left(\varepsilon \hat{\mu}_{a}+\varepsilon^{-1} \hat{\mu}_{s}^{\prime}\right)}=\frac{\varepsilon}{3\left(\varepsilon^{2} \hat{\mu}_{a}+\hat{\mu}_{s}^{\prime}\right)} \approx \frac{\varepsilon}{3 \hat{\mu}_{s}^{\prime}}=\frac{1}{3 \mu_{s}^{\prime}} .
$$

\subsection{Mathematical setting of the diffusion approximation.}

We have seen in the previous sections that the RTE can be approximated by the diffusion equation under the assumptions that the source term is isotropic and the absorption coefficient is small compared to the scattering coefficient. The diffuse photon density, as unknown of the diffusion equation, is a function of space and time. However, in the case where the light source is pulsed at a fixed frequency $\omega>0$, the photon density at point $x$ reads $\mathfrak{R} e(\Phi(x) \exp (i \omega t))$ with a complex-valued function $\Phi$. Then, $\Phi$ is solution to the following time-harmonic diffusion equation

$$
-\operatorname{div}(\kappa \nabla \Phi)+\left(\mu_{a}+\frac{i \omega}{c}\right) \Phi=q .
$$

Here and below, we will define the diffusion coefficient $\kappa$ from formula (10)

$$
\kappa=\frac{1}{3\left(\mu_{a}+\mu_{s}^{\prime}\right)} .
$$

We are interested in the analysis of light propagation in the human head. The propagation domain is thus a bounded regular domain $\Omega \subset \mathbb{R}^{3}$ with boundary $\Gamma=\partial \Omega$, and the optical parametersr $\mu_{a}$ and $\mu_{s}^{\prime}$ are functions of the space variable $\mathbf{x}$. Usually, they are given by piecewise constant functions which take constant values in each tissue of the head. From a mathematically point of view, we will make the assumptions that

$$
\mu_{a}, \mu_{s}^{\prime} \in L^{\infty}(\Omega), m \leq \mu_{a}(\mathbf{x}), \mu_{s}^{\prime}(\mathbf{x}) \leq M \text { for almost every } \mathbf{x} \in \Omega,
$$


where $m$ and $M$ are two strictly positive constants. This implies that the parameters $\left(\kappa, \mu_{a}\right)$ of the diffusion equation belong to the set of admissible parameters

$$
\mathcal{P}=\left\{\left(\kappa, \mu_{a}\right) \in L^{\infty}(\Omega) \times L^{\infty}(\Omega) \mid m \leq \kappa(\mathbf{x}), \mu_{a}(\mathbf{x}) \leq M \text { for almost every } \mathbf{x} \in \Omega\right\},
$$

where we kept the same notations for the (generic) constants $m$ and $M$ as before.

On the boundary $\Gamma$, the diffusion equation is usually completed by a Robin-type boundary condition (see e.g. [5] and references therein) that takes into account the mismatch of the refractive indices between the biological tissues of the head and the surrounding medium filled with air,

$$
\Phi+2 \chi \kappa \partial_{n} \Phi=0 \text { on } \Gamma \text {. }
$$

The parameter $\chi$ is a constant given by the following formula (see [27]):

$$
\chi=\frac{\frac{2}{1-R_{0}}-1+\left|\cos \theta_{c}\right|^{3}}{1-\left|\cos \theta_{c}\right|^{2}} .
$$

Here, $R_{0}=\left(\frac{n / n_{0}-1}{n / n_{0}+1}\right)^{2}$ with $n$ (resp. $\left.n_{0}\right)$ the refractive index of the tissue (resp. of air), and $\theta_{c}=$ $\arcsin \left(n_{0} / n\right)$. Since $n>n_{0}$, we have $0<R_{0}<1$ and $\chi$ can be shown to be strictly positive. Summing up, the complete model for the time-harmonic diffusion approximation reads as follows: find a complex-valued function $\Phi: \Omega \rightarrow \mathbb{C}$ such that

$$
\left\{\begin{aligned}
-\operatorname{div}(\kappa \nabla \Phi)+\left(\mu_{a}+\frac{i \omega}{c}\right) \Phi & =q \text { in } \Omega \\
\Phi+2 \chi \kappa \partial_{n} \Phi & =0 \text { on } \Gamma .
\end{aligned}\right.
$$

The variational formulation of (18) reads

$$
\left\{\begin{array}{l}
\text { Find } \Phi \in H^{1}(\Omega) \text { such that } \\
a(\Phi, \psi)=\ell(\psi) \forall \psi \in H^{1}(\Omega)
\end{array}\right.
$$

where the sesqui-linear form $a(\cdot, \cdot)$ is defined for complex-valued functions of $H^{1}(\Omega)$ by

$$
a(\Phi, \psi)=\int_{\Omega} \kappa \nabla \Phi \cdot \nabla \bar{\psi} d \mathbf{x}+\int_{\Omega}\left(\mu_{a}+\frac{i \omega}{c}\right) \Phi \bar{\psi} d \mathbf{x}+\frac{1}{2 \chi} \int_{\Gamma} \Phi \bar{\psi} d s
$$

and the linear form $\ell(\cdot)$ is defined by

$$
\ell(\psi)=\int_{\Omega} q \bar{\psi} d \mathbf{x}
$$

Theorem 2.1 Assume that the source term q belongs to the space $L^{2}(\Omega)$. Let $\mu_{a}$ and $\mu_{s}^{\prime}$ belong to $L^{\infty}(\Omega)$ such that (15) holds true. Further, let $\chi$ be a strictly positive constant. Then, the variational formulation (19) admits a unique solution $\Phi \in H^{1}(\Omega)$.

The proof follows in a classical way from Lax-Milgram's Theorem since the (complex-valued) sesquilinear form is coercive on $H^{1}(\Omega)$, i.e. there is a constant $\alpha>0$ such that

$$
\mathfrak{R} e(a(\Phi, \Phi)) \geq \alpha\|\Phi\|_{1, \Omega}^{2} \forall \Phi \in H^{1}(\Omega) .
$$

\subsection{Modeling of the source term.}

In diffuse optical tomography or near infra-red spectroscopy, the biological tissue is illuminated by a light source. This light source is usually realized by a laser beam at the surface of the object under examination. One distinguishes three types of devices: continuous wave, frequency-domain and time-resolved sources. In the present study, we focus on frequency-domain source terms as a compromise between the quantity of information contained in the recorded signals and the construction cost of the device.

In view of the assumptions allowing the use of the diffusion approximation, the source term must be isotropic. In the case of a focusing laser beam, the center of the source should therefore be placed at a distance of at least one mean free path, i.e. at $\delta=\frac{1}{\mu_{s}^{\prime}}$. At this distance, the emitted photons have lost the memory of the direction they were coming from and the isotropy condition is satisfied [23] . 
Mathematically, a focusing point source can be described by the Dirac delta $q=\delta_{S}$ where $S \in \Omega$ is the source point. However, the Dirac delta is not a function and the mathematical justification of the model is less straightforward. We refer to [3] where a model problem with Dirac right hand side is set in the functional framework of $L^{p}$-spaces. Another possibility would be to split the solution into a singular part that can be described analytically from the knowledge of the fundamental solution of the Laplacian and a regular part that can be computed numerically. This approach is popular in electro-encephalography (EEG) models where the right hand side contains derivatives of the Dirac delta $[15,29,6]$. In our setting, we choose to model the source term as a Gaussian function with specified beam waist: if $q$ is given by the function

$$
q(\mathbf{x})=a \exp \left(-\frac{\|\mathbf{x}-S\|^{2}}{2 \sigma^{2}}\right),
$$

the beam waist where the field amplitude falls to $1 / e$ is related to $\sigma$ by $w=\sqrt{2} \sigma$.

In comparison to the Delta dirac, the numerical simulations showed more stability of the observed variables for the Gaussian source term.

\subsection{Parameter sets.}

In this section, we discuss the choice of a paramater set among those available in literature. This brings up the question whether the diffusion approximation of the RTE is valid or not for the given parameter set.

Realistic head models usually distinguish up to 5 tissues in the brain: scalp and skull that have similar optical properties, gray matter and white matter, and cerebrospinal fluid (CSF) which has low absorption and scattering coefficients.

The following baseline values correspond to optical properties of the neonatal head tissues at a wavelength of $800 \mathrm{~nm}$. They are taken from [17] and have been used in [13]. The reduced scattering coefficient has been obtained by multiplying $\mu_{s}$ by the factor $(1-g)$ with $g=0.9$ for all tissues. The last column exhibits the ratio between $\mu_{a}$ and $\mu_{s}$ and yields an estimation of the small parameter $\varepsilon$ in the asymptotic expansion described in Section 2.1.2. Indeed, from the assumptions (11), we infer that

$$
\frac{\varepsilon^{2} \mu_{s}}{\mu_{a}}=\frac{\hat{\mu}_{s}}{\hat{\mu}_{a}}=\mathcal{O}(1)
$$

Therefore, the ratio $\mu_{a} / \mu_{s}$ is of order $\mathcal{O}\left(\varepsilon^{2}\right)$ and

$$
\sqrt{\frac{\mu_{a}}{\mu_{s}}} \approx \varepsilon
$$

yields an estimation of the parameter $\varepsilon$. We notice that the ratio in CSF is about one magnitude higher than in scalp or skull. However, the difference is less important compared to gray and white matter.

\begin{tabular}{|c||c|c|c|c|}
\hline Tissue & $\mu_{s}^{\prime}\left[\mathrm{mm}^{-1}\right]$ & $\mu_{a}\left[\mathrm{~mm}^{-1}\right]$ & $\frac{\mu_{a}}{\mu_{s}^{\prime}}$ & $\varepsilon=\sqrt{\frac{\mu_{a}}{\mu_{s}}}$ \\
\hline \hline scalp & 1.9 & 0.018 & 0.0095 & 0.03 \\
skull & 1.6 & 0.016 & 0.01 & 0.03 \\
gray matter & 0.5 & 0.048 & 0.096 & 0.098 \\
white matter & 1.0 & 0.037 & 0.037 & 0.06 \\
$\mathrm{CSF}$ & 0.032 & 0.0041 & 0.128 & 0.11 \\
\hline
\end{tabular}

Table 1: Baseline values for optical parameters of the neonatal head at $800 \mathrm{~nm}$. Anisotropy factor $g=0.9$ for all tissues.

\subsection{Sensitivity analysis of the measurements in presence of inclusions. Nu- merical results.}

In this section we study the impact of the CSF layer on surface measurements in the presence of one inclusion in the inner layer (brain without distinction of white and grey matter). 
The motivation is to analyze the ability of the device to detect intraventricular hemorraghes (IVH) and periventricular leucomalacia (PLM) which are types of brain injury that affect premature infants.

In our first numerical study, the computational domain is a two-dimensional disk of total radius $R_{\Omega}=60[\mathrm{~mm}]$ divided into four concentric layers: two layers, each of thickness $5[\mathrm{~mm}]$, representing respectively the scalp and the skull, a third layer of thickness $2[\mathrm{~mm}]$ filled with CSF, and the inner layer of brain that contains a circular inclusion of radius $15[\mathrm{~mm}]$ (see Figure 1). Since a circular multi-layer model is not able to account for the detailed folds of the cortex, we take the mean between the respective values of white and grey matter for the absorption and scattering coefficients of the brain tissue. Finally, we take $\mu_{a}=0.1\left[\mathrm{~mm}^{-1}\right]$ and $\mu_{s}^{\prime}=3\left[\mathrm{~mm}^{-1}\right]$ in the inclusion. Figure 2 (left picture) shows the difference in the Log-scale between the modulus $\left|\Phi^{\text {pert }}\right|$ of the photon density in presence of the perturbation and the modulus $\left|\Phi^{\text {ref }}\right|$ in the reference configuration without perturbation. One clearly sees that boundary measurements are not able to detect the presence of the inclusion. In comparison, we show the difference between boundary measurements with and without an inclusion if the optical parameters of the second layer are those of skull instead of CSF. In this case, boundary measurements taken on the perturbed configuration contain information about the presence of an inclusion.
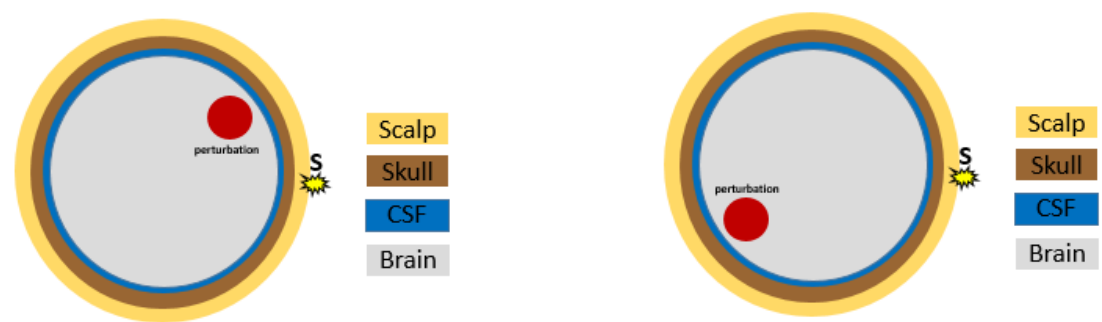

Figure 1: 2D four-layer head model: scalp (yellow), skull (brown), CSF (blue), white/grey matter (grey), with circular inclusion (red). Inclusion near to the source (left). Inclusion opposite to the source (right).
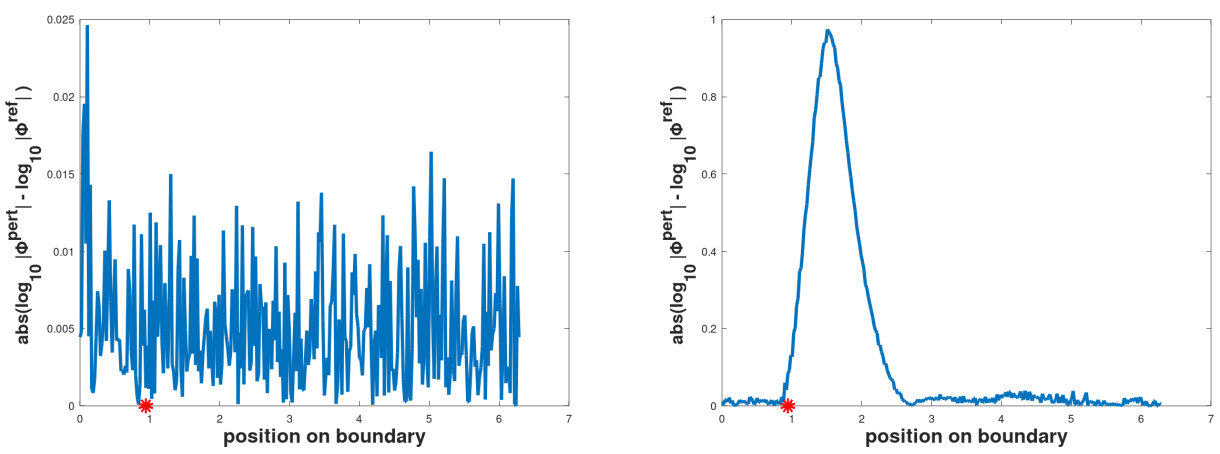

Figure 2: Sensitivity of the boundary measurements to the presence of an inclusion. Diffusion approximation. Third layer: CSF (left), skull (right). Center of perturbation projected on the boundary (red star).

\section{A new homogenized model in the CSF layer.}

\subsection{The problematic case of the CSF layer. State of the art.}

An important number of papers state that the diffusion approximation is not valid in the CSF layer. Indeed, as we have seen in Section 2.5, inclusions in the brain become nearly invisible in the presence of CSF. However, the values of the optical parameters in CSF related in Table 1 attest that the absorption coefficient is still significantly smaller than the scattering coefficient. The ratio $\sqrt{\mu_{a} / \mu_{s}}$ which can be taken as a measure for the scaling parameter $\varepsilon$ in the asymptotic analysis is about 3 times larger in CSF than in skull, but it is still small compared to 1 . A similar ratio is by the way observed in gray matter. 
This indicates that the error that occurs by taking the diffusion approximation instead of the RTE will be more important in CSF and gray matter than in skull or scalp tissues.

A comparative study between RTE and DA [20] attests that for a ratio of $\mu_{a} / \mu_{s}^{\prime}=0.1$ (which is approximatively the case for CSF and for gray matter), the diffusion theory underestimates the photon density by almost 35\%. However, this estimation depends on the distance between the measurement point and the source point which is $5 \mathrm{~cm}$ in the study. Due to the important mean free path $(\mathrm{mfp})$ in CSF (about $3 \mathrm{~cm}$ ), Figure 2 in [20] seems to indicate that the error is less than $5 \%$ at $5 \mathrm{mfp}=15 \mathrm{~cm}$ which covers entirely the neonatal head.

Another study [11] compares the RTE- and DA-model in a circular geometry containing a thin ring of a non- or low-scattering medium. In this study, the DA approximation overestimates the RTE. However, the scattering coefficients of the two models are not the same: $\mu_{s}=0\left[\mathrm{~mm}^{-1}\right]$ for RTE and $\mu_{s}^{\prime}=0.005\left[\mathrm{~mm}^{-1}\right]$ and the absorption coefficient has been taken constant over the whole domain $\left(\mu_{a}=0.025\left[\mathrm{~mm}^{-1}\right]\right)$. These values differ strongly from the baseline optical parameters that we found in the literature (Table 1) which makes an interpretation of the results in the realistic context of the neonatal head difficult. The same paper states that the DA model is not able to reconstruct the optical parameters in the presence of inclusions whenever the thickness of the clear layer exceeds $0.5 \mathrm{~mm}$. This corroborates our results of Section 2.5.

Since the coupling of RTE and DA in a realistic head model is difficult due to non-local boundary conditions on the interfaces [21], our aim is to develop a model based on the only diffusion approximation that is able to deal with CSF. We started from the statement that experimental data contain information about the presence of inclusions in the inner brain tissues, whereas DA together with the optical parameters of Table 1 is obviously not sensitive to these perturbations. We therefore looked for a more sophisticated description of the tissues that does not reduce the CSF layer to a simple homogeneous void region. We follow the idea of [24] to take into account arachnoid trabeculæin CSF, but we apply homogenization in order to substitute the two-phase layer by an equivalent homogeneous layer. This reduces the number of mesh elements and thus the computational cost in a significant manner.

\subsection{Description of the periodic structure.}

Homogenization is mostly applied to media that have periodic microstructure since explicit formula for the equivalent homogeneous medium can be obtained. In this section, we make precise this periodic structure of CSF containing discrete scatterers that we have in mind.

Assume thus a given periodic medium recovered by small cells $Y_{\delta}$ indexed with the parameter $\delta>0$ (Figure 3, right). Let $Y=]-1 / 2,1 / 2\left[{ }^{d}\right.$ be the macroscopic cell of volume 1 . Then, $\left.Y_{\delta} \stackrel{\text { def }}{=} \delta Y=\right]-\delta / 2, \delta / 2\left[{ }^{d}\right.$ denotes the cell at the microscopic scale. For a point $\mathbf{x}$ belonging to a microscopic cell $Y_{\delta}$, the point $\mathbf{y}=\frac{\mathbf{x}}{\delta}$ belongs to $Y$. Now, assume that each cell $Y_{\delta}$ contains two phases (in our case AT surrounded by CSF). A (generic) physical parameter $k_{\delta}$ is thus defined in the following way by a partition of the cell $Y$ into two subdomains $Y^{\mathrm{AT}}$ (containing AT) and $Y^{\mathrm{CSF}}$ (containing CSF),

$$
k_{\delta}(\mathbf{x})=k\left(\frac{\mathbf{x}}{\delta}\right)
$$

where

$$
k(\mathbf{y})= \begin{cases}k^{\mathrm{AT}} & \text { in } Y^{\mathrm{AT}} \\ k^{\mathrm{CSF}} & \text { in } Y^{\mathrm{CSF}}\end{cases}
$$

and $k^{\mathrm{AT}}, k^{\mathrm{CSF}}$ are strictly positive constants. Consequently, if $p=\frac{\operatorname{area}\left(Y^{\mathrm{AT}}\right)}{\operatorname{area}(Y)}$ denotes the proportion of AT in the medium, the mean $<k_{\delta}>$ of the parameter $k_{\delta}$ is given by

$$
<k_{\delta}>=\frac{1}{\operatorname{area}(Y)} \int_{Y} k(\mathbf{y}) d \mathbf{y}=p k^{\mathrm{AT}}+(1-p) k^{\mathrm{CSF}}
$$

\subsection{Homogenization results for the diffusion equation.}

Homogenization theory for partial differential equations (PDE) of diffusion type has been widely studied in the literature (see $[9,26,7]$ and references therein).

If the governing $\mathrm{PDE}$ reads

$$
-\operatorname{div}\left(k_{\delta} \nabla u_{\delta}\right)+\mu_{\delta} u_{\delta}=f \text { in } \Omega,
$$



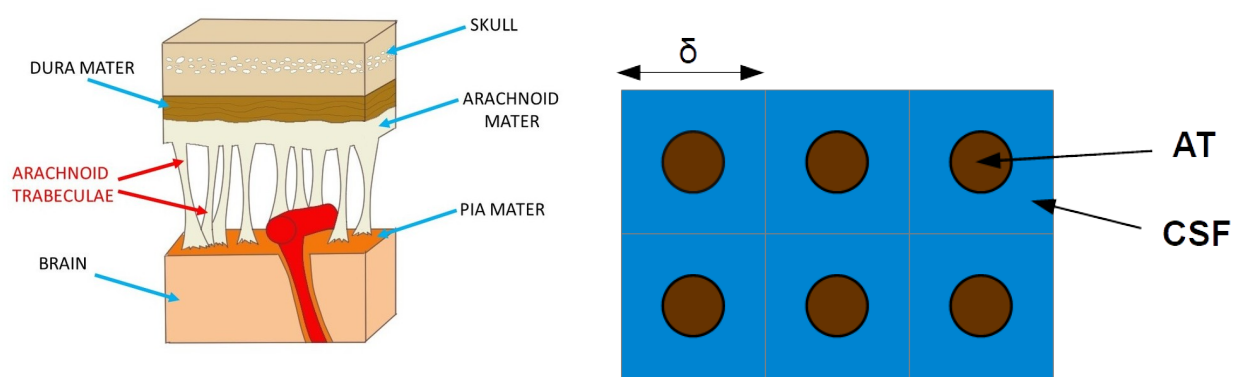

Figure 3: Illustration of a domain with periodic microstructures. Presence of arachnoid trabeculae in CSF (left). Recovering of the domain by microcells $Y_{\delta}$ (right).

with parameter functions $k_{\delta}=k_{\delta}(\mathbf{x})$ and $\mu_{\delta}=\mu_{\delta}(\mathbf{x})$ depending on the space variable $\mathbf{x}$ as described in Section 3.2, the associated homogenized equation is obtained by a two-scale approach [1] and reads

$$
-\operatorname{div}\left(\mathbb{K}^{*} \nabla u\right)+\mu^{*} u=f \text { in } \Omega
$$

where $\mathbb{K}^{*} \in \mathcal{M}_{d}(\mathbb{R})$ is a symmetric matrix and $\mu^{*}=<\mu_{\delta}>$ is the mean of $\mu_{\delta}$. The coefficients of the matrix $\mathbb{K}^{*}$ can be obtained from the following formula

$$
\mathbb{K}_{i j}^{*}=\int_{Y}\left(k(\mathbf{y})+k(\mathbf{y}) \nabla_{y} \chi_{j} \cdot \mathbf{e}_{i}\right) d \mathbf{y}
$$

Here, $\left(\mathbf{e}_{i}\right)_{1, \ldots, d}$ denotes the canonical basis of $\mathbb{R}^{d}$ and $\left(\chi_{j}\right)_{j=1, \ldots, d}$ are the solutions of $d$ auxiliary problems in the macroscopic cell $Y$

It turns out, however, that this approach does not yield a correct description of the presence of discrete AT scatterers in CSF since the asymptotic behavior of the characteristic length scales is not satisfied: indeed, in order to be valid, the mean free path in the background medium should be small compared to the microscopic length scale of the inhomogeneities. In our configuration, the discrete scatterers are generally assumed to have a diameter of $0.1 \mathrm{~mm}$, whereas the mean free path in CSF is about $31 \mathrm{~mm}$ according to the values of Table 1 . Numerically, we see nearly no difference between simulations with pure CSF and the homogenized medium (Figures 2 (left) and 4). The matrix $\mathbb{K}^{*}$ behaves, roughly speaking, as the mean of the diffusion parameter $\kappa$ which undergoes only slight modification in the homogenization process (see Table 2). It can be clearly seen that classical homogenization of the diffusion equation does not yield any improvement on the sensitivity of the measurements to the presence of an inclusion.

\begin{tabular}{|c||c|cccc|}
\hline$p$ & classical DA & 0.10 & 0.15 & 0.20 & 0.30 \\
\hline \hline$\langle\kappa\rangle$ & 9.23 & 8.33 & 7.88 & 7.43 & 6.52 \\
\hline
\end{tabular}

Table 2: Mean value of the diffusion parameter $\kappa$ for different percentages $p$ of AT among CSF.

In the sequel, we thus follow a different approach. We first proceed to homogenization of the RTE and then use the diffusion approximation with the homogenized parameters of the RTE.

\subsection{Homogenization results for the RTE.}

Homogenization of the RTE has been less studied than for the diffusion equation. Here, we use the results of [14, Theorem 3.1] and refer to references therein for an overview of the state of the art.

The following theorem yields the convergence result of the homogenization process in the context of RTE and identifies the optical parameters of the homogenized RTE.

Theorem 3.1 Let $\mu_{a}$ and $\mu_{s}$ be piecewise constant functions on the cell $Y$, defined, respectively, by

$$
\mu_{a}(\mathbf{y})=\left\{\begin{array}{ll}
\mu_{a}^{\mathrm{AT}} & \text { in } Y^{\mathrm{AT}}, \\
\mu_{a}^{\mathrm{CSF}} & \text { in } Y^{\mathrm{CSF}},
\end{array} \quad \text { and } \quad \mu_{s}(\mathbf{y})= \begin{cases}\mu_{s}^{\mathrm{AT}} & \text { in } Y^{\mathrm{AT}}, \\
\mu_{s}^{\mathrm{CSF}} & \text { in } Y^{\mathrm{CSF}},\end{cases}\right.
$$




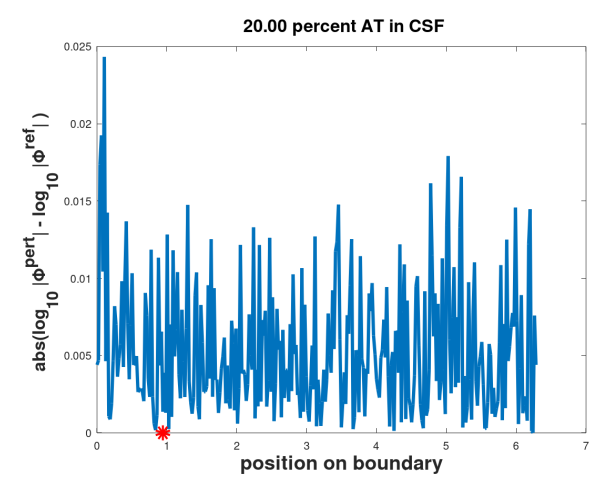

Figure 4: Sensitivity of the boundary measurements to the presence of an inclusion in the inner layer. DA with classical homogenization in the coefficients $\kappa$ and $\mu_{a}$. Center of perturbation projected on the boundary (red star).

where $\mu_{a}^{\mathrm{AT}}, \mu_{a}^{\mathrm{CSF}}\left(\right.$ resp. $\left.\mu_{s}^{\mathrm{AT}}, \mu_{s}^{\mathrm{CSF}}\right)$ are strictly positive constants. For $\delta>0$, let

$$
\mu_{a, \delta}(\mathbf{x})=\mu_{a}\left(\frac{\mathbf{x}}{\delta}\right), \quad \text { and } \quad \mu_{s, \delta}(\mathbf{x})=\mu_{s}\left(\frac{\mathbf{x}}{\delta}\right)
$$

be the associated periodic oscillating functions defined on the microscopic cell $Y_{\delta}$. Assume that the phase function $f\left(\mathbf{s}, \mathbf{s}^{\prime}\right)$ belongs to $L^{\infty}(\mathbb{S} \times \mathbb{S})$ and satisfies

$$
\int_{\mathbb{S}} \int_{\mathbb{S}}\left|f\left(\mathbf{s}, \mathbf{s}^{\prime}\right)-f\left(\mathbf{s}, \mathbf{s}^{\prime}+t\right)\right| d \mathbf{s}^{\prime} \rightarrow 0
$$

when $t \rightarrow 0$ on $\mathbb{S}$. Let $I_{\delta}(t, \mathbf{x}, \mathbf{s})$ denote the photon intensity, solution of the following equation in the domain covered by the microscopic cells $Y_{\delta}$,

$$
\frac{1}{c} \partial_{t} I_{\delta}(t, \mathbf{x}, \mathbf{s})+\mathbf{s} \cdot \nabla I_{\delta}(t, \mathbf{x}, \mathbf{s})+\mu_{t, \delta} I_{\delta}(t, \mathbf{x}, \mathbf{s})=\frac{\mu_{s, \delta}}{4 \pi} \int_{\mathbb{S}} f\left(\mathbf{s}, \mathbf{s}^{\prime}\right) I_{\delta}\left(t, \mathbf{x}, \mathbf{s}^{\prime}\right) d \mathbf{s}^{\prime}+q(t, \mathbf{x}, \mathbf{s})
$$

with initial condition $I^{(0)} \in L^{\infty}\left(\mathbb{R}^{d}, \mathbb{S}\right)$. Then, as $\delta \rightarrow 0, I_{\delta}$ converges weakly- ${ }^{*}$ in $L^{\infty}$ to the solution $I$ of the homogenized RTE,

$$
\frac{1}{c} \partial_{t} I(t, \mathbf{x}, \mathbf{s})+\mathbf{s} \cdot \nabla I(t, \mathbf{x}, \mathbf{s})+\left(\mu_{a}^{*}+\mu_{s}^{*}\right) I(t, \mathbf{x}, \mathbf{s})-\frac{\mu_{s}^{*}}{4 \pi} \int_{\mathbb{S}} f\left(\mathbf{s}, \mathbf{s}^{\prime}\right) I(t, \mathbf{x}, \mathbf{s}) d \mathbf{s}^{\prime}=q(t, \mathbf{x}, \mathbf{s})
$$

with initial condition

$$
I(0, \mathbf{x}, \mathbf{s})=I^{(0)}(\mathbf{x}, \mathbf{s}) \text { a.e. },
$$

and the homogenized coefficients $\mu_{a}^{*}$ and $\mu_{s}^{*}$ are given by the respective mean values:

$$
\mu_{a}^{*}=<\mu_{a}>\quad \text { and } \quad \mu_{s}^{*}=<\mu_{s}>\text {. }
$$

\section{Proof.}

The assumptions on the coefficients enter within the particular example mentioned in [14] where the coefficient $\mu_{t, \delta}=\mu_{a, \delta}+\mu_{s, \delta}$ is independent of the direction variable $\mathbf{s}$ and the collision operator $\mu_{s, \delta}(\mathbf{x}) f\left(\mathbf{s}, \mathbf{s}^{\prime}\right)$ is separated in the variables $\mathbf{x}$ and $\mathbf{s}$. The assumptions of Theorem 3.1 in [14] are thus fulfilled.

It remains to prove that the sequences $\left(\mu_{a, \delta}\right)_{\delta}$ and $\left(\mu_{s, \varepsilon}\right)_{\delta}$ converge weakly-* in $L^{\infty}$ to their respective mean values $\left\langle\mu_{a}>\right.$ and $\left\langle\mu_{s}>\right.$. This is a standard result in functional analysis. For the sake of completeness, we give the proof in Appendix A.

In [14], the governing equation does not contain any source term. However, since in the setting of RTE, the source term $q$ is independent of the oscillating parameter $\delta$, the generalization to the inhomogeneous case is straigthforward and the convergence result keeps true. 


\subsection{The homogenized DA model.}

Our final goal is to use the diffusion approximation in each layer. In order to overcome the lack of validity of DA in the CSF layer, we proposed to take into account the presence of discrete scatterers, the arachnoid trabeculæ. As in [24], we assume that arachnoid trabeculæ have optical properties close to skull. In Table 3, we report the homogenized values for the absorption coefficient $\mu_{a}^{*}$ and the reduced scattering coefficient $\mu_{s}^{\prime, *}$ for different percentages of AT among CSF. Here, the homogenized reduced scattering coefficient is given by $\mu_{s}^{* *}=(1-g) \mu_{s}^{*}$ with an anisotropic factor $g=0.9$ as before. The value of the homogenized diffusion coefficient $\kappa^{*}$ is computed from $\mu_{a}^{*}$ and $\mu_{s}^{\prime *}$ by

$$
\kappa^{*}=\frac{1}{3\left(\mu_{a}^{*}+\mu_{s}^{\prime *}\right)}
$$

\begin{tabular}{|c||c|cccc|}
\hline$p$ & classical DA & 0.10 & 0.15 & 0.20 & 0.30 \\
\hline \hline$\mu_{a}^{*}\left[\mathrm{~mm}^{-1}\right]$ & 0.0041 & 0.0053 & 0.0059 & 0.0065 & 0.0077 \\
$\mu_{s}^{\prime *}\left[\mathrm{~mm}^{-1}\right]$ & 0.032 & 0.1888 & 0.2672 & 0.3256 & 0.5024 \\
\hline$\kappa^{*}$ & 9.23 & 1.72 & 1.22 & 0.95 & 0.65 \\
\hline$\frac{\mu_{a}^{*}}{\mu_{s}^{\prime *}}$ & 0.1281 & 0.0280 & 0.0220 & 0.0188 & 0.0153 \\
$\varepsilon^{*}=\sqrt{\frac{\mu_{a}^{*}}{\mu_{s}^{*}}}$ & 0.1131 & 0.0529 & 0.0469 & 0.0433 & 0.0391 \\
\hline
\end{tabular}

Table 3: Homogenized absorption, scattering and diffusion coefficients for different percentages $p$ of AT among CSF. Baseline values for AT: $\mu_{a}^{\mathrm{AT}}=0.016\left[\mathrm{~mm}^{-1}\right], \mu_{s}^{\mathrm{AT}}=1.6\left[\mathrm{~mm}^{-1}\right]$. Ratio between absorption and reduced scattering coefficient. Scaling factor for DA.

One clearly sees that for all given percentages, the assumption $\mu_{a}^{*} \ll \mu_{s}^{\prime *}$ is satisfied. The diffusion approximation is thus valid in all layers with a scaling factor $\varepsilon^{*}$ that varies between 0.04 and 0.05 compared to 0.03 in scalp and skull.

The new homogenized model for time-harmonic optical tomography then reads: find a complex-values function $\Phi^{*}: \Omega \rightarrow \mathbb{C}$ such that

$$
\left\{\begin{aligned}
-\operatorname{div}\left(\kappa^{*} \nabla \Phi^{*}\right)+\left(\mu_{a}^{*}+\frac{i \omega}{c}\right) \Phi^{*} & =q \text { in } \Omega, \\
\Phi^{*}+2 \chi \kappa^{*} \partial_{n} \Phi^{*} & =0 \text { on } \Gamma,
\end{aligned}\right.
$$

where $\kappa^{*}$ is given by (27) with absorption and scattering coefficients from Table 1 for scalp, scull, grey and white matter, and homogenized values for $\mu_{a}^{*}$ and $\mu_{s}^{\prime *}$ according to Table 3.

\subsection{Sensitivity analysis of the measurements in presence of inclusions. Nu- merical results for the new homogenized DA model.}

In this section, we analyze the behavior of our new DA model in the presence of a perturbation. We test several geometric configurations and different proportions of AT among CSF. We then represent the difference in the log-scale between the modulus of the perturbed solution $\left|\Phi^{*, p e r t}\right|$ and the reference solution $\left|\Phi^{*, r e f}\right|$ at the boundary.

In the first example, the perturbation is located in the first quadrant of the inner disc near to the light source at a distance of $2[\mathrm{~mm}]$ from the brain/CSF interface (cf. Figure 1, left). We clearly see in Figure 5 (upper line) that even for only $10 \%$ AT among CSF, the sensitivity to the presence of the inclusion is improved, and we observe a link between the projection of the inclusion's center on the boundary and the difference between the reference and the perturbed solution.

In the second example (Figure 5, lower line), the perturbation is located in the third quadrant opposite to the light source, again at a distance of $2[\mathrm{~mm}]$ from the interface. The same observations as for the first test case can be made: the presence of AT among CSF improves sensitivity to the presence of the perturbation. Furthermore, the peak of the difference yields a better approximation of the projection of the center as in the first example where the perturbation was located near the source term.

Next, we consider the new homogenized model with 10\% AT among CSF for a perturbation located at different distances from the brain/CSF interface (cf. Figure 6). As expected, sensitivity improves with 

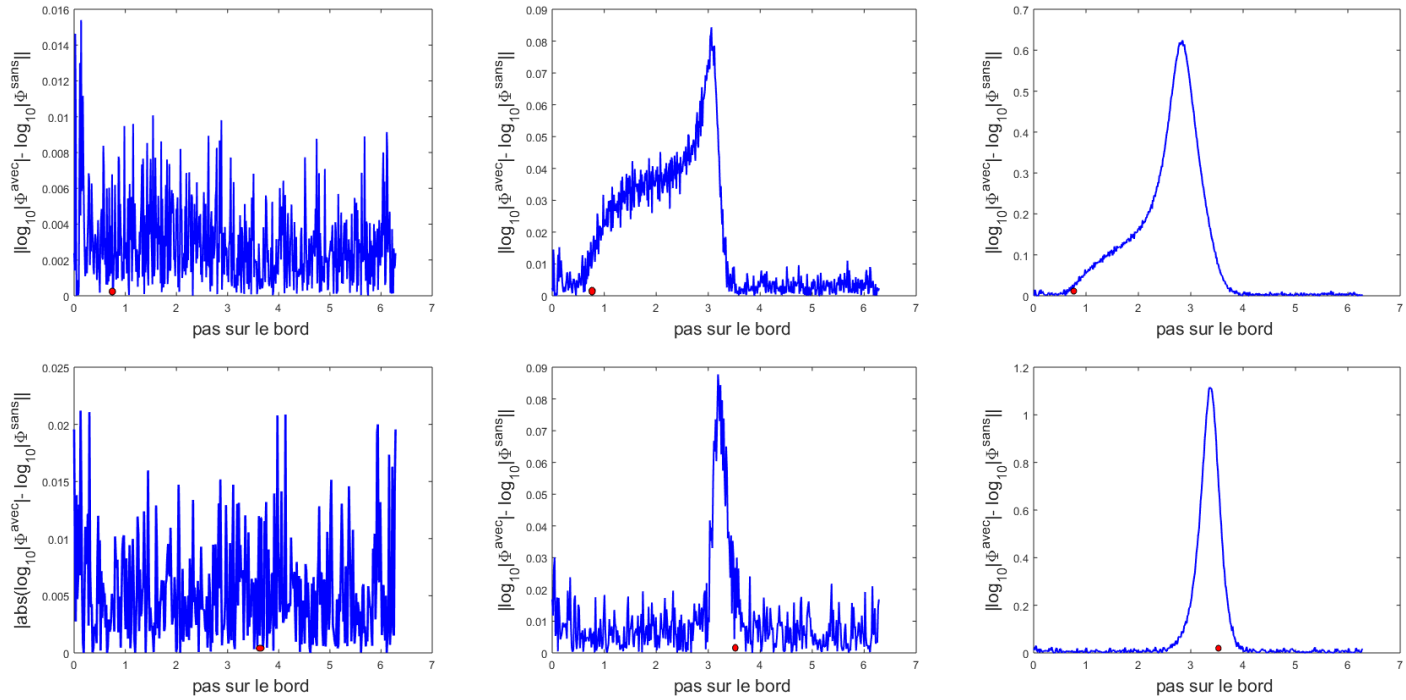

Figure 5: Sensitivity of the boundary measurements to the presence of an inclusion. Classical DA model (left column). New homogenized DA model. 10\% of AT among CSF (center), $20 \%$ of AT among CSF (right column). Upper line: inclusion near to the source (cf. Fig. 1 - left). Lower line: inclusion opposite to the source (cf. Fig. 1 - right). Center of inclusion projected on the boundary (red star).
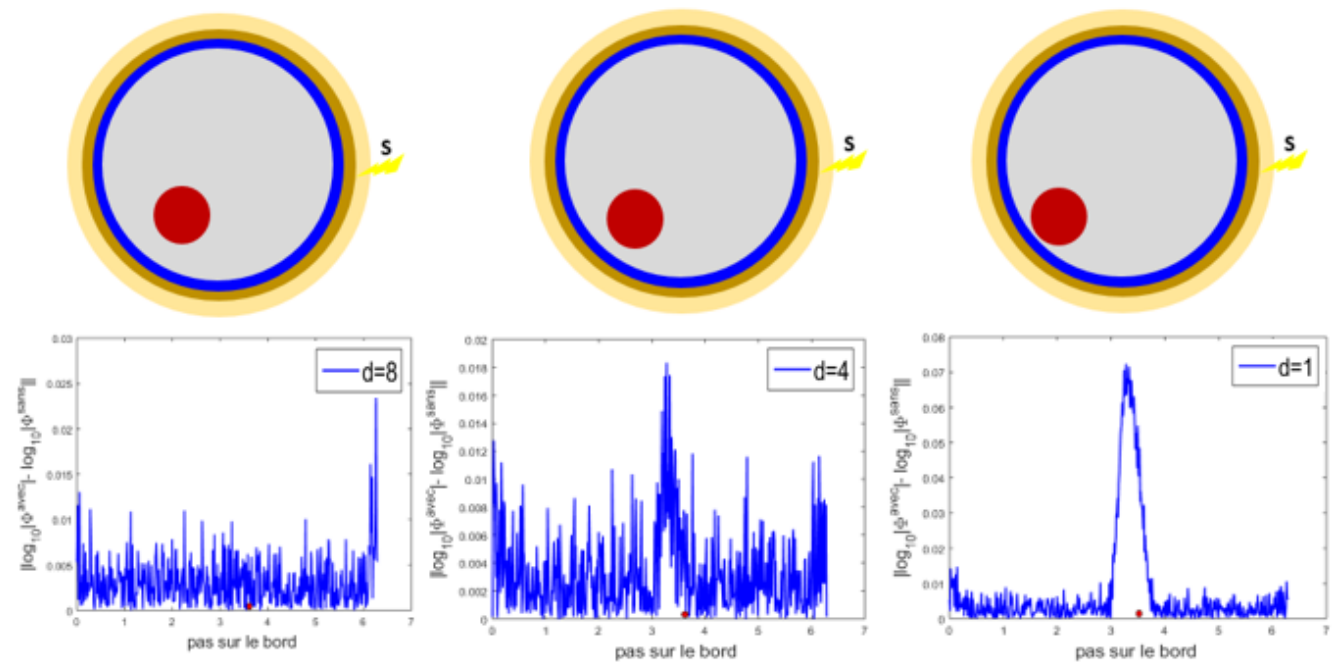

Figure 6: Sensitivity of the boundary measurements to the presence of an inclusion located at different distances from the CSF/brain interface: 8 [mm] (left), 4 [mm] (center), 1 [mm] (right). New homogenized DA model with $10 \%$ AT among CSF. Center of perturbation projected on the boundary (red star).

decreasing distance. But the new homogenized model allows for the detecting perturbations that are located deeper in the brain than the classical one.

Similar observations can be made in 3D where we considered a four-layer spherical domain (scalp, skull, CSF, brain). The boundary values of the difference between the perturbed and the reference solution are represented on the equirectangular projection of the sphere (cf. Figure 7 ). In the case where the perturbation is situated near the source point, its visual localization from the boundary data seems to be difficult, but we clearly see that the presence of the inclusion becomes visible if we take into account $10 \%$ AT among CSF. For a perturbation located far from the source point, information about its location can be retrieved from the surface plot. 

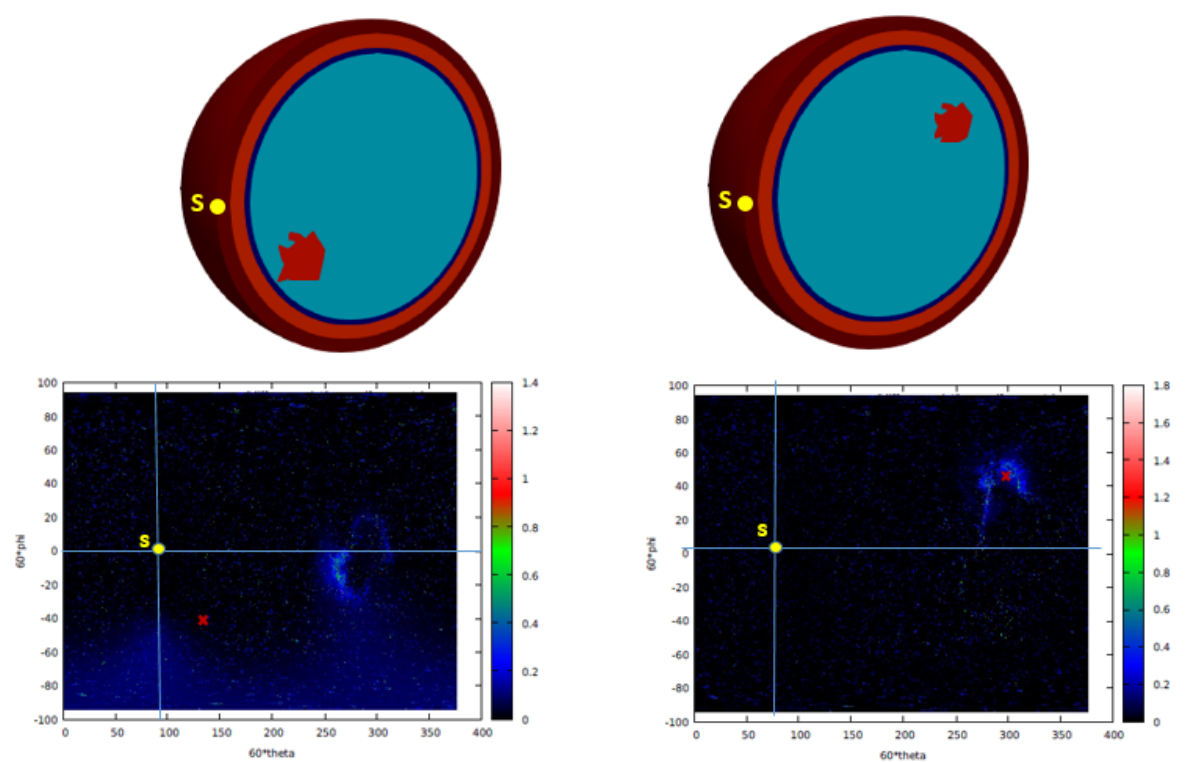

Figure 7: Equirectangular projection of the difference between the perturbed and the reference solution (lower line). New homogenized model with 10\% AT among CSF. Perturbation near the source (left column). Perturbation far from the source (right column).

\section{Sensitivity analysis via the Gâteaux derivative.}

For better reading, we denote in the sequel by $\Phi$ (instead of $\left.\Phi^{*}\right)$ the solution of the new DA model for a given proportion $p$ of AT among CSF. In the same way, the parameters of the new model are denoted by $\kappa$ and $\mu_{a}$ instead of $\kappa^{*}$ and $\mu_{a}^{*}$. Notice that for $p=0$, we recover the classical model that does not take into account the presence of arachnoid trabeculæ.

Sensitivity describes the variation of the solution to a problem according to the variation of the parameters of the problem. In the present context, we aim to study the sensitivity of the solution to the diffusion equation $\Phi=\Phi[\mu]$ with respect to variations of the parameters $\mu=\left(\kappa, \mu_{a}\right)$.

Mathematically, this concept of sensitivity is formulated in terms of the directional derivative. More precisely, for a direction $\delta \mu=\left[\delta \kappa, \delta \mu_{a}\right] \in L^{\infty}(\Omega) \times L^{\infty}(\Omega)$, the derivative of $\Phi$ at $\mu \in \mathcal{P}$ in the direction $\delta \mu$ is given by

$$
\Phi^{1}[\mu]=\lim _{h \rightarrow 0} \frac{\Phi[\mu+h \delta \mu]-\Phi[\mu]}{h},
$$

if the limit exists in $H^{1}(\Omega)$. Here, $\mathcal{P}$ denotes the set of admissible parameters defined in (16).

\subsection{The sensitivity equation.}

Let $\mu=\left(\kappa, \mu_{a}\right)$ be a fixed couple of parameters in the set $\mathcal{P}$. The derivative $\Phi^{1}$ defined by $(29)$ can be shown to be the solution of a variational problem that involves the same sesqui-linear form as the original problem:

Proposition 4.1 Let $\delta \mu=\left(\delta \kappa, \delta \mu_{a}\right)$ be a direction in $L^{\infty}(\Omega) \times L^{\infty}(\Omega)$. For a given couple of parameters $\mu \in \mathcal{P}, \Phi^{1}[\mu]$ is the unique solution in $H^{1}(\Omega)$ to the variational equation

$$
a\left(\Phi^{1}[\mu], \Psi\right)=-\int_{\Omega} \delta \kappa \nabla \Phi[\mu] \cdot \nabla \bar{\Psi} d \mathbf{x}-\int_{\Omega} \delta \mu_{a} \Phi[\mu] \bar{\Psi} d \mathbf{x} \forall \Psi \in H^{1}(\Omega)
$$

where $\Phi[\mu] \in H^{1}(\Omega)$ is the solution of problem

$$
a(\Phi[\mu], \Psi)=\ell(\Psi) \forall \Psi \in H^{1}(\Omega) .
$$

Here, $a(\cdot, \cdot)$ is the sesqui-linear form defined by (20) with parameters $\kappa$ and $\mu_{a}$.

The proof of Proposition 4.1 is straightforward and can be adapted e.g. from the results in [6] where sensitivity with respect to the electrical conductivity has been studied in the context of electro-encephalography. 


\subsection{The adjoint problem.}

For a given couple of parmeters $\mu=\left(\kappa, \mu_{a}\right) \in \mathcal{P}$, let $M[\mu]$ denote the measurement at a given detector situated at position $\mathbf{r}_{d} \in \Gamma$. If $M[\mu]$ depends on the solution $\Phi[\mu]$ of the diffusion equation in a linear way, define the linear form $b: H^{1}(\Omega) \rightarrow \mathbb{C}$ such that $b(\Phi[\mu])=M[\mu]$. Consequently, the derivative of $M$ at $\mu$ in the direction $\delta \mu$ is given by $M^{1}[\mu]=b\left(\Phi^{1}[\mu]\right)$. Now, define the adjoint problem of (31) by

$$
\left\{\begin{array}{l}
\text { Find } p[\mu] \in H^{1}(\Omega) \text { such that } \\
a(q, p[\mu])=b(q) \forall q \in H^{1}(\Omega)
\end{array}\right.
$$

Obviously, (32) admits a unique solution since $\mu=\left(\kappa, \mu_{a}\right)$ belongs to the set of admissible parameters $\mathcal{P}$ and $b(\cdot)$ is continuous. Now, taking $q=\Phi^{1}[\mu]$ in (32) and $\psi=p[\mu]$ in the sensitivity equation (30), we see that

$$
M^{1}[\mu]=b\left(\Phi^{1}[\mu]\right)=-\int_{\Omega} \delta \kappa \nabla \Phi[\mu] \cdot \nabla \overline{p[\mu]} d \mathbf{x}-\int_{\Omega} \delta \mu_{a} \Phi[\mu] \overline{p[\mu]} d \mathbf{x} .
$$

Now, we discretize the parameters with the help of finite elements of type P0 on a mesh $\mathcal{T}_{h}=\left(T_{\ell}\right)_{\ell=1: L}$ of $L$ elements. These piecewise constant finite elements are well suited to represent functions of the space $L^{\infty}$. The canonical basis functions of the associated discretization space are the characteristic functions $\chi_{\ell}$ of the mesh elements. The decomposition of the parameters in the basis $\left(\chi_{\ell}\right)_{\ell}$ together with $(33)$ yields

$$
M^{1}[\mu]=\sum_{\ell=1}^{L}(\delta \kappa)_{\ell} J_{\ell}^{\kappa}+\left(\delta \mu_{a}\right)_{\ell} J_{\ell}^{\mu_{a}}
$$

where

$$
\begin{aligned}
J_{\ell}^{\mu_{a}} & =-\int_{T_{\ell}} \Phi[\mu] \overline{p[\mu]} d \mathbf{x} \\
J_{\ell}^{\kappa} & =-\int_{T_{\ell}} \nabla \Phi[\mu] \cdot \nabla \overline{p[\mu]} d \mathbf{x}
\end{aligned}
$$

for $\ell=1: L$. The coefficient $J_{\ell}^{\kappa}$ thus represents the sensitivity of the measurement at a detector $\mathbf{r}_{d}$ with respect to a small variation of the parameter $\kappa$ in the mesh element $T_{\ell}$.

\subsection{Numerical results.}

In this section, we show some numerical results of sensitivity. To this end, the measurement operator $M$ has to be precised.

A possible definition is the pointwise measurement $M[\mu]=\Phi\left(\mathbf{r}_{d}\right)$. Notice however that this example does not define a continuous linear form on $H^{1}(\Omega)$ since functions in $H^{1}$ are not well defined at a single point. Indeed, in this case the adjoint problem is equivalent to a boundary value problem with Dirac right hand side and other techniques should be used to get a rigorous solution theory. Formally, we recognize here the well known reciprocity principle in optical tomography which states that the photon density at a point $\mathbf{r}_{d}$ due to a source situated at $\mathbf{r}_{s}$ is the same as the one at $\mathbf{r}_{s}$ due to a source situated at $\mathbf{r}_{d}$ (see e.g. [4]). Roughly speaking, this means that in the adjoint problem, the detector plays the role of the source term in the adjoint problem and vice versa.

Here, we define the measurement as the photon density on a small neighborhood of the detector located at $\mathbf{r}_{d}$,

$$
M[\mu]=\int_{\Gamma} \Phi[\mu](s) g\left(\mathbf{r}_{d}-s\right) d s
$$

where $g$ is a (given) Gaussian function centered at 0 . Then, the associated linear form $b$ is well defined on $H^{1}(\Omega)$ by

$$
b(q)=\int_{\Gamma} q g\left(\mathbf{r}_{d}-s\right) d s
$$

The sensitivity with respect to $\mu_{a}$ and $\kappa$ is then computed respectively through formulas (34) and (35). One may observe that the measurements are more sensitive to changes in the banana-like region linking the detector to the source position if the new homogenized DA model is used (see Figure 8) and increases with the proportion of AT among CSF. One further notices that measurements are more sensitive to changes in the absorption coefficient $\mu_{a}$ than in the diffusion coefficient $\kappa$. 

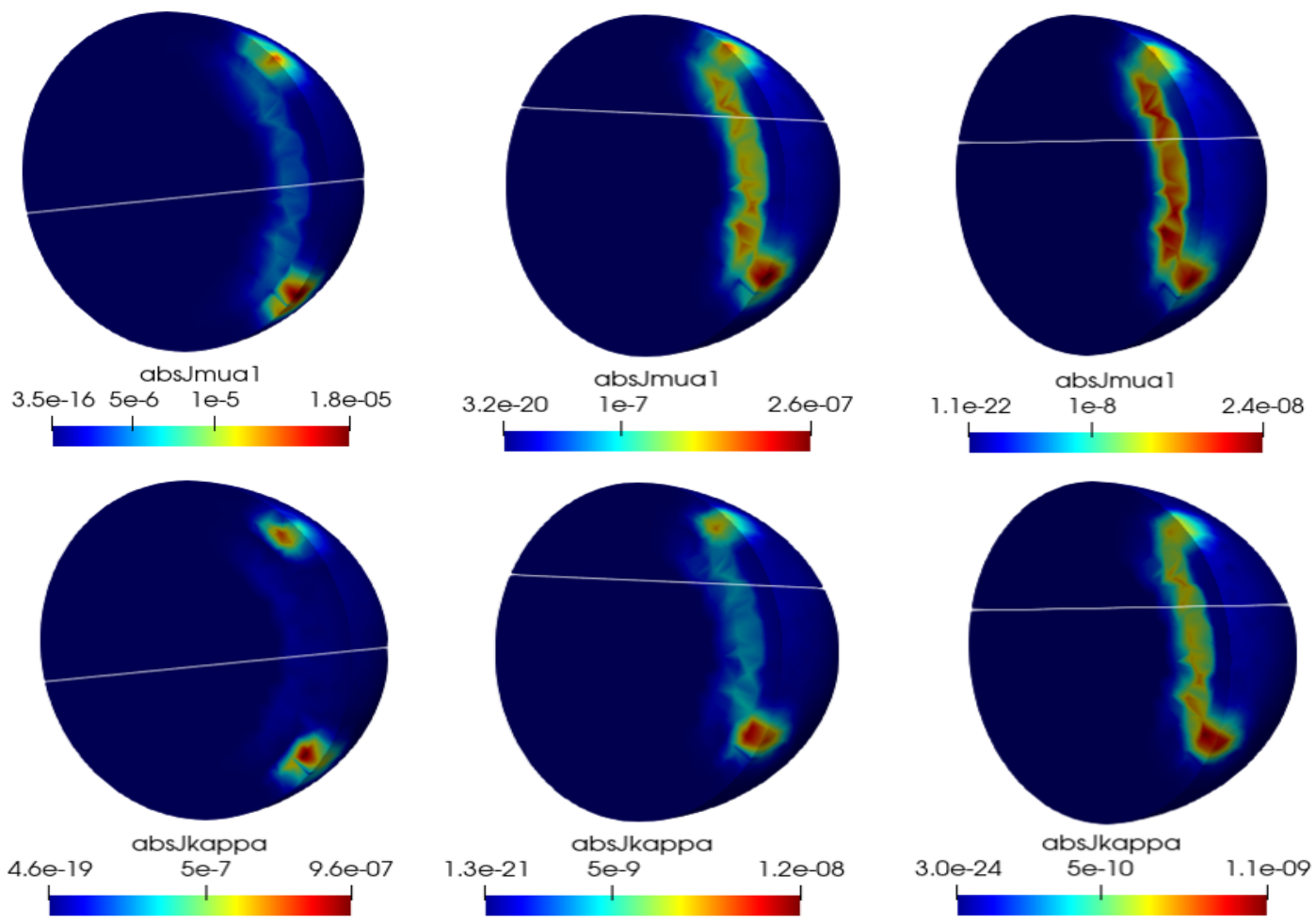

Figure 8: Sensitivity with respect to $\mu_{a}$ (upper line) and $\kappa$ (lower line) for a given source/detector couple in a four-layer spherical model. Classical DA model (left column). New homogenized DA model with $10 \%$ AT among CSF (center) and 20\% AT among CSF (right column).

\section{Concluding remarks.}

We presented a new model for optical tomography in the neonatal brain that takes into account the presence of arachnoid trabeculæ in cerebrospinal fluid. The new model is based on the diffusion approximation of the homogenized radiative transfer equation in the CSF layer (see Figure 9). The homogenization process has been rigorously justified by applying existing homogenization results for transport equations. The equivalent homogenized CSF coefficients allow approximation of the RTE by the diffusion equation. Numerical simulations in 2D and 3D show the improved sensitivity of the new model with respect to the presence of perturbations in the brain layer. The new model has the same computational complexity as the classical diffusion approximation and is thus well suited to be used in inverse parameter reconstruction for which first promising results have been obtained. This item will be adressed in futur work.

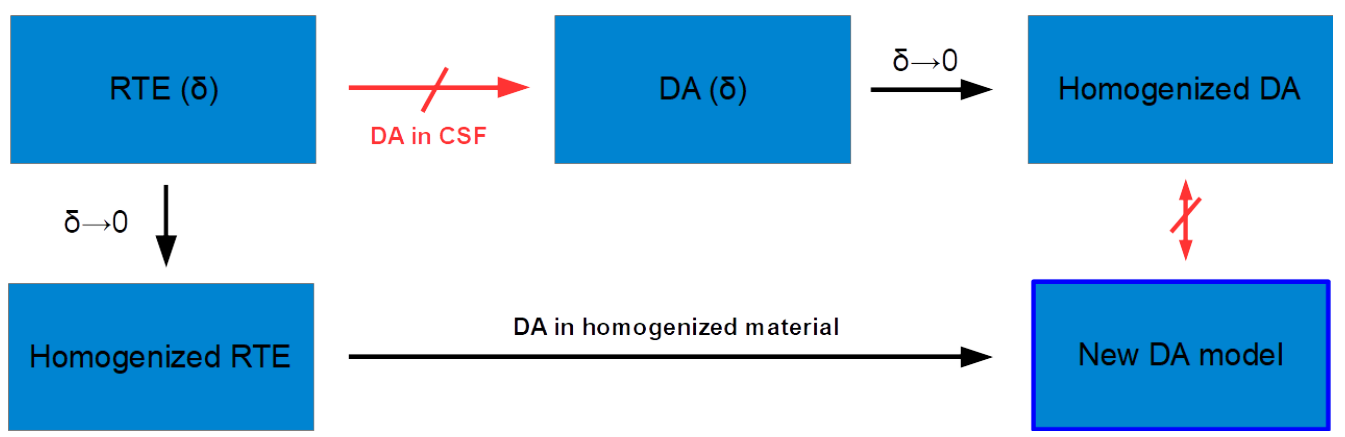

Figure 9: How to obtain the new DA model. 


\section{A Weak-* convergence to the mean value.}

Lemma A.1 Let $w \in L^{\infty}\left(\mathbb{R}^{d}\right)$ be a periodic function satisfying

$$
w\left(\mathbf{x}+\mathbf{e}_{i}\right)=w(x) \text { for almost every } \mathbf{x} \in \mathbb{R}^{d}, \forall i=1, \ldots, d
$$

where $\left(\mathbf{e}_{i}\right)_{i=1, \ldots, d}$ denotes the canonical basis of $\mathbb{R}^{d}$. Let $\left.Y=\right] 0,1\left[{ }^{d}\right.$ be the unit cell in $\mathbb{R}^{d}$ and $W=$ $\int_{Y} w(\mathbf{x}) d \mathbf{x}$ the mean value of the function $w$. For $n \in \mathbb{N}$, define the sequence $\left(w_{n}\right)_{n \in \mathbb{N}}$ by $w_{n}(\mathbf{x})=w(n \mathbf{x})$. Then,

$$
w_{n} \rightarrow W \text { weak- }^{*} \text { in } L^{\infty}\left(\mathbb{R}^{n}\right) .
$$

Proof. We will give the proof in two dimensions for the sake of readability. Without loss of generality, we assume that $W=0$. Let $\left.E=] a_{1}, b_{1} \times\right] a_{2}, b_{2}[$. We have

$$
\int_{E} w(n \mathbf{x}) d \mathbf{x}=\frac{1}{n^{2}} \int_{n a_{2}}^{n b_{2}} \int_{n a_{1}}^{n b_{1}} w(\mathbf{y}) d \mathbf{y} .
$$

Now, for $i=1,2$, let $N_{i}=\left[n b_{i}-n a_{i}\right]$ be the integer part of $n b_{i}-n a_{i}$. Hence,

$$
n b_{i}-1 \leq n a_{i}+N_{i} \leq n b_{i} .
$$

We split the integrals into a sum of integrals over intervals of length 1 and a rest:

$$
\begin{aligned}
\frac{1}{n^{2}} \int_{n a_{2}}^{n b_{2}} \int_{n a_{1}}^{n b_{1}} w(\mathbf{y}) d \mathbf{y} . & =\frac{1}{n^{2}} \sum_{k_{2}=0}^{N_{2}-1}\left(\sum_{k_{1}=0}^{N_{1}-1} \int_{n a_{2}+k_{2}}^{n a_{2}+k_{2}+1} \int_{n a_{1}+k_{1}}^{n a_{1}+k_{1}+1} w(y) d y\right) \\
& +\frac{1}{n^{2}} \int_{n a_{2}}^{n a_{2}+N_{2}} \int_{n a_{1}+N_{1}}^{n b_{1}} w(\mathbf{y}) d \mathbf{y} \\
& +\frac{1}{n^{2}} \int_{n a_{2}+N_{2}}^{n b_{2}} \int_{n a_{1}+N_{1}}^{n b_{1}} w(\mathbf{y}) d \mathbf{y}
\end{aligned}
$$

Now, the first term in the above expression vanishes since $w$ has 0 mean value and is 1 -periodic. The second term can be majored as follows

$$
\frac{1}{n^{2}} \int_{n a_{2}}^{n a_{2}+N_{2}} \int_{n a_{1}+N_{1}}^{n b_{1}} w(\mathbf{y}) d \mathbf{y} \leq \frac{N_{2}\left(n b_{1}-n a_{1}-N_{1}\right)}{n^{2}}\|w\|_{L^{\infty}(Y)}
$$

and tends to zero since $\frac{N_{2}}{n^{2}} \leq \frac{b_{2}-a_{2}}{n}$ and $n b_{1}-n_{1}-N_{1} \leq 1$. In the same way, the third term is of order $\mathcal{O}\left(\frac{1}{n^{2}}\right)$ and tends to zero as $n \rightarrow \infty$. This shows that

$$
\lim _{n \rightarrow \infty} \int_{E} w_{n} \xi d \mathbf{x}=0
$$

for any step function $\xi$. By density, this holds true for any function $\xi \in L^{1}\left(\mathbb{R}^{d}\right)$ and thus

$$
w_{n} \rightarrow 0 \text { weak-* }^{*} \text { in } L^{\infty} .
$$

\section{B Proof of the homogenization result for the RTE}

In [2], the following linear Boltzmann equation is studied:

$$
\partial_{t} f(t, \mathbf{x}, \mathbf{v})+\mathbf{v} \cdot \nabla_{x} f(t, \mathbf{x}, \mathbf{v})+a(t, \mathbf{x}, \mathbf{v}) f(t, \mathbf{x}, \mathbf{v})=\int_{\mathbb{R}^{n}} k(t, \mathbf{x}, \mathbf{v}, \mathbf{w}) f(t, \mathbf{x}, \mathbf{w}) d \mu(\mathbf{w})+q(t, \mathbf{x}, \mathbf{v})
$$

completed by the initial condition

$$
f(0, \cdot, \cdot)=f^{\text {in }}
$$


Notice that $\mu$ denotes a general positive Radon measure on $\mathbb{R}^{n}$. In particular, this covers the case of the surface measure on the unit sphere $\mathbb{S}$,

$$
\int_{\mathbb{R}^{n}} \Phi d \mu=\int_{\mathbb{S}} \Phi(\mathbf{s}) d \mathbf{s}
$$

We make the following assumptions:

$$
\begin{aligned}
& a \geq 0, a \in \mathcal{C}_{b}\left([0, T] \times \mathbb{R}^{n} \times \mathbb{R}^{n}\right) \\
& k \geq 0, \quad k \in \mathcal{C}_{b}\left([0, T] \times \mathbb{R}^{n} \times \mathbb{R}^{n} \times \mathbb{R}^{n}\right) \\
& q \in \mathcal{C}_{b}\left([0, T] \times \mathbb{R}^{n} \times \mathbb{R}^{n}\right) \\
& f^{\text {in }} \in \mathcal{C}_{b}\left(\mathbb{R}^{n} \times \mathbb{R}^{n}\right) .
\end{aligned}
$$

Under the assumptions (39), the Cauchy problem (37)-(38) admits a unique solution $f \in \mathcal{C}_{b}\left([0, T], \mathbb{R}^{n}, \mathbb{R}^{n}\right)$ (Theorem 3.1.2 in [2]). If, in addition, $f^{\text {in }} \geq 0$ and $q \geq 0$, then the solution $f$ of (37)-(38) satisfies $f \geq 0$ and

$$
f(t, \mathbf{x}, \mathbf{v}) \leq\left(\left\|f^{\text {in }}\right\|_{L^{\infty}}+T\|q\|_{L^{\infty}}\right) e^{D t}
$$

where

$$
D=\sup _{t, \mathbf{x}, \mathbf{v}} \int_{\mathbb{R}^{n}} k(t, \mathbf{x}, \mathbf{v}, \mathbf{w}) d \mu(\mathbf{w})
$$

(Proposition 3.1.4. and following remark in [2]). In the case where the measure is the surface measure on the unit sphere and the kernel $k$ is bounded by a constant $M$, we have

$$
D=2 \pi^{2} M .
$$

The same results can be obtained for data belonging to $L^{\infty}$ instead of $\mathcal{C}_{b}[2]$.

Now, let $I_{\delta}(t, \mathbf{x}, \mathbf{s})$ denote the solution of $(24)$ with initial condition $I^{(0)} \in L^{\infty}\left(\mathbb{R}^{d} \times \mathbb{S}\right)$. From $(40)$, it follows that the sequence $\left(I_{\delta}\right)_{\delta}$ is bounded in $L^{\infty}\left([0, T] \times \mathbb{R}^{d} \times \mathbb{S}\right)$. Hence, there is a sub-sequence, denoted by $\left(I_{\delta_{n}}\right)_{n}$, and $I \in L^{\infty}\left([0, T] \times \mathbb{R}^{d} \times \mathbb{S}\right)$ such that

$$
I_{\delta_{n}} \stackrel{*}{\rightarrow} I \text { weakly-* in } L^{\infty} \text {. }
$$

It follows from equation $(24)$ that $\Psi_{\delta}=\partial_{t} I_{\delta}+\mathbf{s} \cdot \nabla_{x} I_{\delta}$ is also bounded in $L^{\infty}\left([0, T] \times \mathbb{R}^{d} \times \mathbb{S}\right)$ and a sub-sequence converges thus weakly-* in $L^{\infty}$ to a limit $\Psi$ :,

$$
\Psi_{\delta_{n}} \stackrel{*}{\rightarrow} \Psi \text { weakly-*} \text { in } L^{\infty} .
$$

Since the weak*-convergence in $L^{\infty}$ implies convergence in the sense of distributions $-\mathcal{D}\left([0, T] \times \mathbb{R}^{d} \times \mathbb{S}\right) \subset$ $L^{1}\left([0, T] \times \mathbb{R}^{d} \times \mathbb{S}\right)$ - we infer that $\Psi=\partial_{t} I+\mathbf{s} \cdot \nabla_{x} I$ a.e. in $[0, T] \times \mathbb{R}^{d} \times \mathbb{S}$.

In [14] (Prop. 2.3), the velocity averaging method is stated in $L^{1}$. However, it keeps true in $L_{\text {loc }}^{1}$ as well under the same conditions on the measure (see $[18,19])$. We thus need to show that $I_{\delta} \rightarrow I$ weakly in $L_{\text {loc }}^{1}\left(\mathbb{R} \times \mathbb{R}^{d} \times \mathbb{S}\right)$. But the dual space of $L_{\text {loc }}^{1}$ can be identified with the subspace $L_{c}^{\infty}$ of functions in $L^{\infty}$ that have bounded essential support. Then, let $v \in L_{c}^{\infty}\left(\mathbb{R} \times \mathbb{R}^{d} \times \mathbb{S}\right)$. Since the essential support of $v$ is bounded, $v$ belongs to $L^{1}\left(\mathbb{R} \times \mathbb{R}^{d} \times \mathbb{S}\right)$ and it follows from the weakly-* convergence of $\left(I_{\delta_{n}}\right)$ to $I$ that

$$
\int I_{\delta_{n}} v \rightarrow \int I v
$$

This proves the weak convergence of $\left(I_{\delta_{n}}\right)$ in $L_{\text {loc }}^{1}\left(\mathbb{R} \times \mathbb{R}^{d} \times \mathbb{S}\right)$. We prove in the same way that $\Psi_{\delta_{n}} \rightarrow \Psi$ weakly in $L_{\text {loc }}^{1}$.

The result of velocity averaging then states that the sequence

$$
\int_{\mathbb{S}} I_{\delta}(t, \mathbf{x}, \mathbf{s}) \chi(t, \mathbf{x}, \mathbf{s}) d \mathbf{s} \longrightarrow \int_{\mathbb{S}} I(t, \mathbf{x}, \mathbf{s}) \chi(t, \mathbf{x}, \mathbf{s}) d \mathbf{s} \quad \text { strongly in } L^{1}\left(\mathbb{R} \times \mathbb{R}^{d}\right)
$$

for any test function $\chi \in \mathcal{D}\left(\mathbb{R} \times \mathbb{R}^{d} \times \mathbb{S}\right)$. Indeed, the surface measure $\mu=d \mathbf{s}$ on the unit sphere satisfies obviously the condition

$$
\mu(\{\mathbf{s} \in \mathbb{S} \mid \tau+\mathbf{s} \cdot \mathbf{x}=0\})=0
$$

for any $\tau \in \mathbb{R}$ and $\mathbf{x} \in \mathbb{R}^{d} \backslash\{0\}$ since the set $\{\mathbf{s} \in \mathbb{S} \mid \tau+\mathbf{s} \cdot \mathbf{x}=0\}$ is the intersection of the unit sphere and the plane with normal vector $\mathbf{x}$, thus a circle on the unit sphere which has vanishing surface measure. 
We aim to apply Proposition 2.1 of [14] with $a_{n}=I_{\delta_{n}}-I$ and $b_{n}=\mu_{t, \delta_{n}}-\mu_{t}^{*}$ The sequence $b_{n}=\mu_{t, \delta_{n}}-\mu_{t}^{*}$ is independent from the direction $\mathbf{s}$ and thus satisfies in a trivial way

$$
\int_{\mathbb{S}} \int_{|\mathbf{x}| \leq R} \sup _{\left|\mathbf{s}-\mathbf{s}^{\prime}\right|<\varepsilon}\left|b_{n}(\mathbf{x}, \mathbf{s})-b_{n}\left(\mathbf{x}, \mathbf{s}^{\prime}\right)\right| d \mathbf{x} d \mathbf{s} \rightarrow 0
$$

uniformly in $n$ as $\varepsilon \rightarrow 0$. We therefore obtain

$$
\mu_{s, \delta_{n}} I_{\delta_{n}} \rightarrow \mu_{s}^{*} I \text { in } \mathcal{D}^{\prime}\left(\mathbb{R} \times \mathbb{R}^{d} \times \mathbb{S}\right) .
$$

Next, we let $b_{n}\left(\mathbf{x}, \mathbf{s}, \mathbf{s}^{\prime}\right)=\left(\mu_{s, \delta_{n}}(\mathbf{x})-\mu_{s}^{*}\right) f\left(\mathbf{s}, \mathbf{s}^{\prime}\right)$. Since the phase function $f$ is bounded by assumption, we have

$$
\begin{aligned}
\int_{|\mathbf{x}| \leq R} & \int_{\mathbb{S}} \int_{\mathbb{S}\left|\mathbf{s}^{\prime}-\mathbf{s}^{\prime \prime}\right|<\varepsilon} \sup _{n}\left|b_{n}\left(\mathbf{x}, \mathbf{s}, \mathbf{s}^{\prime}\right)-b_{n}\left(\mathbf{x}, \mathbf{s}, \mathbf{s}^{\prime \prime}\right)\right| d \mathbf{s}^{\prime} d \mathbf{s} d \mathbf{x} \\
= & \left(\int_{|\mathbf{x}| \leq R}\left|\mu_{s, \delta_{n}}(\mathbf{x})-\mu_{s}^{*}\right| d \mathbf{x}\right)\left(\int_{\mathbb{S}} \int_{\mathbb{S}\left|\mathbf{s}^{\prime}-\mathbf{s}^{\prime \prime}\right|<\varepsilon}\left|f\left(\mathbf{s}, \mathbf{s}^{\prime}\right)-f\left(\mathbf{x}, \mathbf{s}, \mathbf{s}^{\prime \prime}\right)\right| d \mathbf{s}^{\prime} d \mathbf{s}\right) \\
& \leq C \int_{|\mathbf{x}| \leq R}\left|\mu_{s, \delta_{n}}(\mathbf{x})-\mu_{s}^{*}\right| d \mathbf{x} \\
& \rightarrow 0
\end{aligned}
$$

uniformly in $n$ as $\varepsilon \rightarrow 0$. Indeed, the characteristic function $\chi_{\{|\mathbf{x}| \leq R\}}$ belongs to $L^{1}\left(\mathbb{R}^{d}\right)$ and the integral on the space variable $\mathbf{x}$ thus converges since $\mu_{s, \delta_{n}} \stackrel{*}{\rightarrow} \mu_{s}^{*}$ weakly-* in $L^{\infty}\left(\mathbb{R}^{d}\right)$.

We thus get, applying once more Proposition 2.1 of [14], that

$$
\mu_{t, \delta_{n}} I_{\delta_{n}} \rightarrow \mu_{t}^{*} I \text { in } \mathcal{D}^{\prime}\left(\mathbb{R} \times \mathbb{R}^{d} \times \mathbb{S} \times \mathbb{S}\right) .
$$

From (44), it follows easily that

$$
\int_{\mathbb{S}} \mu_{s, \delta_{n}}(\mathbf{x}) f\left(\mathbf{s}, \mathbf{s}^{\prime}\right) I_{\delta_{n}}\left(t, \mathbf{x}, \mathbf{s}^{\prime}\right) d \mathbf{s}^{\prime} \rightarrow \int_{\mathbb{S}} \mu_{s}(\mathbf{x}) f\left(\mathbf{s}, \mathbf{s}^{\prime}\right) I\left(t, \mathbf{x}, \mathbf{s}^{\prime}\right) d \mathbf{s}^{\prime}
$$

in $\mathcal{D}^{\prime}\left(\mathbb{R} \times \mathbb{R}^{d} \times \mathbb{S}\right)$. Hence, the limit $I$ satisfies the homogenized RTE (25) as well as the initial condition which is independent from $\delta$. This completes the proof.

\section{References}

[1] G. Allaire, Homogenization and two-scale convergence, SIAM J. Math. Anal. 23(6) (1992), pp. 1482 1518.

[2] G. Allaire, X. Blanc, J.-B. Després, and F. Golse, Transport et diffusion, Ed. Ecole Polytechnique, 2019.

[3] T. Apel, O. Benedix, D. Sirch, and B. Vexler, A priori mesh grading for an elliptic problem with Dirac right-hand side, SIAM J. Numer. Anal. 49(3) (2011), pp. 992-1005.

[4] S.R. Arridge, Optical tomography in medical imaging, Inverse Problems 15 (1999), p. R41-R93.

[5] S.R. Arridge and J. Schotland, Optical tomography: forward and inverse problems, Inverse Problems 25(12) (2009), doi:10.1088/0266-5611/25/12/123010.

[6] H. Azizollahi, M. Darbas, M. Diallo, A. El Badia, and S. Lohrengel, EEG in neonates: forward modeling and sensitivity analysis with respect to variations of the conductivity, Math. Biosci. Eng., 15(4) (2018), pp. 905-932.

[7] N. Bakhvalov and G. Panasenko, Homogenization: averaging processes in periodic media, Math. Appl., Vol. 36, Kluwer Academic Publishers, Dordrecht, 1990.

[8] Z. Belhachmi, G. Doll, C. Prud'homme, Christophe, and M. Torregrossa, The direct Robin boundary value parabolic system of time-resolved diffuse optical tomography with fluorescence. J. Math. Study 52 (2019), pp. 341-358. 
[9] A. Bensoussan, J. L. Lions, and G. Papanicolaou, Asymptotic Analysis for Periodic Structures, North-Holland, Amsterdam, 1978.

[10] K. Czuprynski, J. Eichholz, and W. Han, Numerical analysis of the energy-dependent radiative transfer equation, IMA J. Numer. Anal. 39(3) (2019), pp. 1529-1562.

[11] H. Dehghani, D.T. Delpy, and S.R. Arridge, Photon migration in non-scattering tissue and the effects on image reconstruction, Phys. Med. Biol. 44 (1999), pp. 2897-2906.

[12] H. Dehghani, M. E. Eames, P. K. Yalavarthy, S. C. Davis, Near infrared optical tomography using NIRFAST: Algorithm for numerical model and image reconstruction Commun Numer Methods Eng. 25(6) (2008), pp. 711-732.

[13] M. Dehaes, K. Kazemi, M. Pélégrini-Issac, R. Grebe, H. Benali, and F. Wallois, Quantitative Effect of the Neonatal Fontanel on Synthetic Near Infrared Spectroscopy Measurements, Human brain Mapping 34 (2013), pp. 878-889.

[14] L. Dumas and F. Golse, Homogenization of transport equations, SIMA J. Appl. Math. 60(4) (2000), pp. $1447-1470$.

[15] A. El Badia and T. Ha Duong, An inverse source problem in potential analysis, Inverse Problems, 16 (2000), 651-663.

[16] M. Fournier, M. Mahmoudzadeh, K. Kazemi, G. Kongolo, G. Dehaene-Lambertz, R. Grebe, F. Wallois, Realistic head model design and 3D brain imaging of NIRS signals using audio stimuli on preterm neonates for intra-ventricular hemorrhage diagnosis, MICCAI 2012 15, pp. 172-179. doi: 10.1007/978-3-642-33454-2_22.

[17] Y. Fukui, Y. Ajichi, and E. Okada, Monte Carlo prediction of near-infrared light propagation in realistic adult and neonatal head models., Appl. Optics 42 (2003), pp. 2881-2887.

[18] F. Golse, P.-L. Lions, B. Perthame, and R. Sentis, Regularity of the moments of the solution of a transport equation, J. Funct. Anal., 76 (1988), pp. 434-460.

[19] F. Golse and L. Saint-Raymond, Velocity averaging in $L^{1}$ for the transport equation, C. R. Acad. Sci. Paris, Ser. I, 334 (2002), pp. 557-562.

[20] A.H. Hielscher, R.E. Alcouffe, and R. L. Barbour, Comparison of finite-difference transport and diffusion calculations for photon migration in homogeneous and heterogeneous tissues, Phys. Med. Biol. 43 (1998), pp. 1285-1302.

[21] N. Hyvonen, Analysis of optical tomography with non-scattering regions, Proc. Edinburgh Math. Soc. 45(2002), pp. 257-276.

[22] A. Kim and I.V. Prokhorov, Theoretical and numerical analysis of an initial-boundary value problem for the radiative transfer equation with Fresnel matching conditions, Comput. Math. Math. Phys. 58(5) (2018), pp. 735749.

[23] B. Montcel, Tomographie optique diffuse résolue en temps: Applications fonctionnelles en neuroscience., PHD thesis, Université Louis Pasteur Strasbourg I, France, 2005.

[24] E. Okada and D.T. Delpy, Near-infrared light propagation in an adult head model. I. Modeling of low-level scattering in the cerebrospinal fluid layer., Appl. Optics 42(16) (2003), pp. 2906-2914.

[25] J. Riley, H. Dehghani, M. Schweiger, S.R. Arridge, J. Ripoll, and M. Nieto-Vesperinas, 3D optical tomography in the presence of void regions, Optics Express 7(13) (2000), pp. 462-467.

[26] E. Sanchez-Palencia, Nonhomogeneous media and vibration theory, Lecture Notes in Phys. 127, Springer, New York, 1980.

[27] M. Schweiger, S. R. Arridge, M. Hiraoka, and D. T. Delpy, The finite element model for the propagation of light in scattering media: Boundary and source conditions, Med. Phys. 22(1995), pp. 17791792 . 
[28] A.V. Smirnov, M.V. Klibanov, and Loc H. Nguyen, On an inverse source problem for the full radiative transfer equation with incomplete data, SIAM J. Sci. Comput. 41(5) (2019), pp. B929-B952.

[29] C. H. Wolters, H. Köstler, C. Möller, J. Härdtlein, L. Grasedyck and W. Hackbusch, Numerical mathematics of the subtraction approach for the modeling of a current dipole in EEG source reconstruction using finite element head models, SIAM J. Sci. Comput., 30 (2007), pp. 24-45.

[30] M. Zhang, J. Cheng, J. Qiu, High order positivity-preserving discontinuous Galerkin schemes for radiative transfer equations on triangular meshes., J. Comput. Phys. 397 (2019), 108811. 